\title{
An acceleration technique for fixed point iterative methods
}

\author{
Oana Bumbariu
}




\title{
AN ACCELERATION TECHNIQUE FOR SLOWLY CONVERGENT FIXED POINT ITERATIVE METHODS
}

\author{
OANA BUMBARIU
}

Received 21 October, 2011

\begin{abstract}
In this paper we present a new acceleration method for accelerating some numerical sequences of successive approximations that arises in solving certain nonlinear equations. We give a convergence result and some numerical examples to illustrate the abilities of the proposed new acceleration technique.
\end{abstract}

2000 Mathematics Subject Classification: 47H10; 65B99

Keywords: fixed point, Picard iterations, Aitken's $\Delta^{2}$ process, order of convergence

\section{INTRODUCTION}

In the last decade an important domain of numerical analysis was the development and the improvement of several extrapolation algorithms. By an extrapolation process a scalar sequence $\left\{S_{n}\right\}$, convergent to $S$, is transformed into a new sequence $\left\{T_{n}\right\}$, through a sequence transformation $T:\left\{S_{n}\right\} \rightarrow\left\{T_{n}\right\}$. It is said that the transformation $T$ accelerates the convergence speed of the sequence $\left\{S_{n}\right\}$ if it is satisfying the following relation

$$
\lim _{n \rightarrow \infty} \frac{T_{n}-S}{S_{n}-S}=0
$$

In this case we say that $\left\{T_{n}\right\}$ converges faster to $S$, than $\left\{S_{n}\right\}$.

The aim of this paper is twofold: first, to present a new acceleration algorithm inspired from one of the many representations of Aitken's iterated $\Delta^{2}$ process for which we give a convergence result and secondly, to perform with this technique an empirical study for the sequences arising in solving nonlinear equations for some of the test functions that can be found in literature.

\section{THE NEW ACCELERATION METHOD}

Consider a nonlinear equation

$$
f(x)=0
$$


which is equivalently written as a fixed point problem

$$
g(x)=x
$$

where $g:[a, b] \rightarrow[a, b]$ is the iteration function.

We assume that $\alpha$ is the unique root of (2.1) in the interval $[a, b]$ and let $x_{0} \in[a, b]$ be an initial approximation sufficiently close to $\alpha$. Under appropriate assumptions on $f$ (and therefore on $g$ ), the Picard iteration

$$
x_{n+1}=g\left(x_{n}\right), \quad n=0,1, \ldots
$$

converges to the (unique) fixed point $x^{*}$ of $g$, which is the (unique) solution of (2.1) in the interval $[a, b]$.

In what follows we remind the concept of linear convergence that can be found in Berinde [3] and the concept of convergence order, also called $Q$-order of convergence that can be found in monographs by Măruşter [17] and Ortega and Rheinboldt [19].

Definition 1. [9] Let us assume that the sequence $\left\{S_{n}\right\}$, which converges to some limit $S$, satisfies

$$
\lim _{n \rightarrow \infty} \frac{S_{n+1}-S}{S_{n}-S}=\lambda
$$

If $\lambda \neq 1$ holds, we say that the sequence $\left\{S_{n}\right\}$ converges linearly, and if $\lambda=1$ holds, we say that $\left\{S_{n}\right\}$ converges logarithmically.

Let $\left\{x_{n}\right\} \in \mathbb{R}$ be a sequence of real numbers convergent to $\alpha \in \mathbb{R}$ (which is obtained by iterating a fixed point equation).

Definition 2. [17],[19] Let $\left\{x_{n}\right\}$ converge to $\alpha$. If there exist an integer constant $p$, and a real positive constant $C$ such that

$$
\lim _{n \rightarrow \infty}\left|\frac{x_{n+1}-\alpha}{\left(x_{n}-\alpha\right)^{p}}\right|=C
$$

then $p$ is called the order and $C$ the constant of convergence.

It is well known that the convergence order of the Picard iteration is generally linear, see Berinde [3], so the method (2.3) converges slowly to the fixed point $x^{*}$. In order to improve the convergence speed of (2.3) we must use a convergence acceleration process. In the sequel we will present the most important ones. The most popular sequence acceleration method, for a sequence that converges slowly, is Aitken's $\Delta^{2}$ process. The method was given by A.C. Aitken in 1926 [1], who used it to accelerate the convergence of Bernoulli's method for computing the dominant zero of a polynomial. The method is given by

$$
A_{1}^{(n)}=S_{n}-\frac{\left(S_{n+1}-S_{n}\right)^{2}}{S_{n+2}-2 S_{n+1}+S_{n}}=S_{n}-\frac{\left[\Delta S_{n}\right]^{2}}{\Delta^{2} S_{n}}, n=0,1, \ldots,
$$

where $\left\{S_{n}\right\}$ is the sequence to be accelerated and $\Delta$ denotes the forward difference operator, $\Delta S_{n}=S_{n+1}-S_{n}$ and $\Delta^{2} S_{n}=\Delta S_{n+1}-\Delta S_{n}=S_{n+2}-2 S_{n+1}+S_{n}$. As it 
is known, Aitken's $\Delta^{2}$ algorithm is based on a recursive computation, in which each term of the sequence is determined by three previous terms by a simple arithmetic, which implies that Aitken's $\Delta^{2}$ process is a transformation of order $l=2$.

Discussion about Aitken's $\Delta^{2}$ process can be found in books by Lubkin [15], Shanks [20], Thuker [22], [23], Cordellier [10], Weniger [25]. Even a generalization to the vector case was discussed by MacLeod [16]. Modifications and generalizations of Aitken's $\Delta^{2}$ process were suggested by Drummond [11] and Jamieson and O' Beirne [13]. The properties of Aitken's $\Delta^{2}$ process were studied by Baker and GravesMorries, Brezinski [2], [7], [8], Walz [24], Wimp [26]. Those properties are:

(i) Aitken's $\Delta^{2}$ process accelerates linear convergence.

(ii) Aitken's $\Delta^{2}$ process is regular but not accelerative for logarithmically convergent sequences.

The convergence power for Aitken's $\Delta^{2}$ process can be increased by iterating it, yielding the following nonlinear recursive scheme

$$
A_{k+1}^{(n)}=A_{k}^{(n)}-\frac{\left[\Delta A_{k}^{(n)}\right]^{2}}{\Delta^{2} A_{k}^{(n)}}, \quad A_{0}^{(n)}=S_{n} \quad n, k \in \mathbb{N}_{0},
$$

where $\Delta A_{k}^{(n)}=A_{k}^{(n+1)}-A_{k}^{(n)}$ and $\Delta^{2} A_{k}^{(n)}=\Delta A_{k}^{(n+1)}-\Delta A_{k}^{(n)}=A_{k}^{(n+2)}-2 A_{k}^{(n+1)}+$ $A_{k}^{(n)}$, the forward difference operator $\Delta$ acts only upon the superscript $n$ and not upon the subscript $k$. In the case of Aitken's iterated $\Delta^{2}$ process every $A_{k}^{(n)}$ term of the sequence is determined by a recursive formula which requires $2 k+1$ elements, consequently, $A_{k}^{(n)}$ is a transformation of order $l=2 k$.

Like Aitken's $\Delta^{2}$ process, the iterated form has many different expressions, one of them being given by the next formula

$$
A_{k+1}^{(n)}=A_{k}^{(n+1)}-\frac{\left[\Delta A_{k}^{(n)}\right]\left[\Delta A_{k}^{(n+1)}\right]}{\Delta^{2} A_{k}^{(n)}}, A_{0}^{(n)}=S_{n} \quad n, k \in \mathbb{N}_{0} .
$$

Discussions about Aitken's iterated $\Delta^{2}$ process were done in [21], [25], although about its theoretical properties very little seems to be known.

Some other important sequences transformations were obtain by Wynn [27] in 1956, this algorithm is called Wynn's $\epsilon$-algorithm; by Brezinski [6] in 1971, which is an improvement of Wynn's $\epsilon$-algorithm, also called the $\theta$-algorithm and by Levin [14] in 1973.

Our new algorithm we introduce in this paper is similar to expression (2.6). The new proposed acceleration technique is given by

$$
B_{k+1}^{(n)}=B_{k}^{(n+3)}-\frac{\left[\bar{\Delta} B_{k}^{(n+1)}\right]\left[\Delta B_{k}^{(n+2)}\right]}{\bar{\Delta} B_{k}^{(n+1)}-\bar{\Delta} B_{k}^{(n)}}, \quad n, k \in \mathbb{N}
$$


where $B_{0}^{(n)}=S_{n},\left\{S_{n}\right\}$ is the sequence to be accelerated, $\Delta$, the forward difference operator, has been defined before and we denote by $\bar{\Delta}$, the forward difference operator with two steps, $\bar{\Delta} B_{k}^{(n)}=B_{k}^{(n+2)}-B_{k}^{(n)}$. Like in the case of the Aitken's iterated $\Delta^{2}$ process the difference operators, $\Delta$ and $\bar{\Delta}$, act only upon the superscript $n$ and not upon the subscript $k$.

In the sequel we will give conditions on the $B_{i}^{\prime} s$ which ensure that $\lim _{n \rightarrow \infty} B_{k}^{(n)}=S$.

Theorem 1. If $\lim _{n \rightarrow \infty} S_{n}=S$, and if $\forall i, \exists b_{i} \neq 1$ such that $\lim _{n \rightarrow \infty} \frac{B_{i}^{(n+1)}}{B_{i}^{(n)}}=b_{i}$, for $\forall i \neq j, \quad b_{i} \neq b_{j}$. Then $\forall k$ we have $\lim _{n \rightarrow \infty} B_{k}^{(n)}=S$.

Proof. For $k=0$ we have

$$
B_{1}^{(n)}=B_{0}^{(n+3)}-\frac{\left(B_{0}^{(n+3)}-B_{0}^{(n+1)}\right)\left(B_{0}^{(n+3)}-B_{0}^{(n+2)}\right)}{\left(B_{0}^{(n+3)}-B_{0}^{(n+1)}\right)-\left(B_{0}^{(n+2)}-B_{0}^{(n)}\right)},
$$

where $B_{0}^{(n)}=S_{n}$.

Multiplying with $\frac{B_{0}^{(n+2)}}{B_{0}^{(n+2)}}$ we get

$$
B_{1}^{(n)}=B_{0}^{(n+3)}-\frac{\frac{B_{0}^{(n+3)}}{B_{0}^{(n+2)}}-\frac{B_{0}^{(n+1)}}{B_{0}^{(n+2)}}}{\frac{B_{0}^{(n+3)}}{B_{0}^{(n+2)}}-\frac{B_{0}^{(n+1)}}{B_{0}^{(n+2)}}-1+\frac{B_{0}^{(n)}}{B_{0}^{(n+2)}}}\left(B_{0}^{(n+3)}-B_{0}^{(n+2)}\right) .
$$

Taking the limit when $n$ tends to infinity we obtain

$$
\lim _{n \rightarrow \infty} B_{1}^{(n)}=\lim _{n \rightarrow \infty} B_{0}^{(n+3)}-\frac{b_{0}-\frac{1}{b_{0}}}{b_{0}-\frac{1}{b_{0}}-1+\frac{1}{b_{0}^{2}}} \lim _{n \rightarrow \infty}\left(B_{0}^{(n+3)}-B_{0}^{(n+2)}\right)
$$

from where it results

$$
\lim _{n \rightarrow \infty} B_{1}^{(n)}=\lim _{n \rightarrow \infty} B_{0}^{(n+3)}-\frac{b_{0}}{b_{0}-1} \lim _{n \rightarrow \infty}\left(B_{0}^{(n+3)}-B_{0}^{(n+2)}\right),
$$

where $b_{0} \neq 1$ and $B_{0}^{(n)}=S_{n}$. Hence

$$
\lim _{n \rightarrow \infty} B_{1}^{(n)}=\lim _{n \rightarrow \infty} S_{n+3}-\frac{b_{0}}{b_{0}-1} \lim _{n \rightarrow \infty}\left(S_{n+3}-S_{n+2}\right),
$$

because $\lim _{n \rightarrow \infty} S_{n}=S$ we obtain $\lim _{n \rightarrow \infty} B_{1}^{(n)}=S$.

Suppose that $\lim _{n \rightarrow \infty} B_{k-1}^{(n)}=S$ we prove that $\lim _{n \rightarrow \infty} B_{k}^{(n)}=S$.

$$
B_{k}^{(n)}=B_{k-1}^{(n+3)}-\frac{\left(B_{k-1}^{(n+3)}-B_{k-1}^{(n+1)}\right)\left(B_{k-1}^{(n+3)}-B_{k-1}^{(n+2)}\right)}{\left(B_{k-1}^{(n+3)}-B_{k-1}^{(n+1)}\right)-\left(B_{k-1}^{(n+2)}-B_{k-1}^{(n)}\right)}
$$


Multiplying with $\frac{B_{k-1}^{(n+2)}}{B_{k-1}^{(n+2)}}$ we obtain

$$
B_{k}^{(n)}=B_{k-1}^{(n+3)}-\frac{\frac{B_{k-1}^{(n+3)}}{B_{k-1}^{(n+2)}}-\frac{B_{k-1}^{(n+1)}}{B_{k-1}^{(n+2)}}}{\frac{B_{k-1}^{(n+3)}}{B_{k-1}^{(n+2)}}-\frac{B_{k-1}^{(n+1)}}{B_{k-1}^{(n+2)}}-1+\frac{B_{k-1}^{(n)}}{B_{k-1}^{(n+2)}}}\left(B_{k-1}^{(n+3)}-B_{k-1}^{(n+2)}\right) .
$$

Taking the limit when $n$ tends to infinity we obtain

$$
\lim _{n \rightarrow \infty} B_{k}^{(n)}=\lim _{n \rightarrow \infty} B_{k-1}^{(n+3)}-\frac{b_{k-1}-\frac{1}{b_{k-1}}}{b_{k-1}-\frac{1}{b_{k-1}}-1+\frac{1}{b_{k-1}^{2}}} \lim _{n \rightarrow \infty}\left(B_{0}^{(n+3)}-B_{0}^{(n+2)}\right),
$$

hence there

$$
\lim _{n \rightarrow \infty} B_{k}^{(n)}=\lim _{n \rightarrow \infty} B_{k-1}^{(n+3)}-\frac{b_{k-1}}{b_{k-1}-1} \lim _{n \rightarrow \infty}\left(B_{k-1}^{(n+3)}-B_{k-1}^{(n+2)}\right)
$$

where $b_{k-1} \neq 1$ and $B_{k-1}^{(n)}=S_{n}$ we obtain $\lim _{n \rightarrow \text { infty }} B_{k}^{(n)}=S$.

Theorem 2. If the conditions of Theorem 1 are satisfied and if $\lim _{n \rightarrow \infty} \frac{B_{k-1}^{(n+1)}-S}{B_{k-1}^{(n)}-S}=b_{k}$. Then $\left\{B_{k}^{(n)}\right\}$ converges to $S$ faster than $\left\{B_{k-1}^{(n)}\right\}$, when $n$ tends to infinity, that is

$$
\lim _{n \rightarrow \infty} \frac{B_{k}^{(n)}-S}{B_{k-1}^{(n)}-S}=0
$$

Moreover if $b_{k} \neq 0,\left\{B_{k}^{(n)}\right\}$ converges to $S$ faster than $\left\{B_{k-1}^{(n+1)}\right\}$.

Proof. We prove the first part of theorem.

$$
\frac{B_{k}^{(n)}-S}{B_{k-1}^{(n)}-S}=\frac{B_{k-1}^{(n+3)}-S-\frac{\left(B_{k-1}^{(n+3)}-B_{k-1}^{(n+1)}\right)\left(B_{k-1}^{(n+3)}-B_{k-1}^{(n+2)}\right)}{\left(B_{k-1}^{(n+3)}-B_{k-1}^{(n+1)}\right)-\left(B_{k-1}^{(n+2)}-B_{k-1}^{(n)}\right)}}{B_{k-1}^{(n)}-S},
$$

adding $S-S$ in each parenthesis and separating into two fractions we obtain

$$
\begin{aligned}
& \frac{B_{k}^{(n)}-S}{B_{k-1}^{(n)}-S}=\frac{B_{k-1}^{(n+3)}-S}{B_{k-1}^{(n)}-S}- \\
& -\frac{\left[\left(B_{k-1}^{(n+3)}-S\right)-\left(B_{k-1}^{(n+1)}-S\right)\right]\left[\left(B_{k-1}^{(n+3)}-S\right)-\left(B_{k-1}^{(n+2)}-S\right)\right]}{\left\{\left[\left(B_{k-1}^{(n+3)}-S\right)-\left(B_{k-1}^{(n+1)}-S\right)\right]-\left[\left(B_{k-1}^{(n+2)}-S\right)-\left(B_{k-1}^{(n)}-S\right)\right]\right\}\left(B_{k-1}^{(n)}-S\right)} .
\end{aligned}
$$


Multiplying with $\frac{B_{k-1}^{(n+2)}-S}{B_{k-1}^{(n+2)}-S}$ it follows that

$$
\begin{gathered}
\frac{B_{k}^{(n)}-S}{B_{k-1}^{(n)}-S} \\
=\frac{B_{k-1}^{(n+3)}-S}{B_{k-1}^{(n+2)}-S} \cdot \frac{B_{k-1}^{(n+2)}-S}{B_{k-1}^{(n)}-S}-\frac{\left[\frac{B_{k-1}^{(n+3)}-S}{B_{k-1}^{(n+2)}-S}-\frac{B_{k-1}^{(n+1)}-S}{B_{k-1}^{(n+2)}-S}\right]\left[\frac{B_{k-1}^{(n+3)}-S}{B_{k-1}^{(n+2)}-S}-1\right]}{\left[\frac{B_{k-1}^{(n+3)}-S}{B_{k-1}^{(n+2)}-S}-\frac{B_{k-1}^{(n+1)}-S}{B_{k-1}^{(n+2)}-S}-1+\frac{B_{k-1}^{(n)}-S}{B_{k-1}^{(n+2)}-S}\right] \frac{B_{k-1}^{(n)}-S}{B_{k-1}^{(n+2)}-S}} .
\end{gathered}
$$

Taking the limit when $n$ tends to infinity we get

$$
\lim _{n \rightarrow \infty} \frac{B_{k}^{(n)}-S}{B_{k-1}^{(n)}-S}=b_{k}^{3}-\frac{\left(b_{k}-\frac{1}{b_{k}}\right)\left(b_{k}-1\right)}{\left(b_{k}-\frac{1}{b_{k}}-1+\frac{1}{b_{k}^{2}}\right) \frac{1}{b_{k}^{2}}},
$$

from where it follows

$$
\lim _{n \rightarrow \infty} \frac{B_{k}^{(n)}-S}{B_{k-1}^{(n)}-S}=b_{k}^{3}-b_{k}^{3}=0 .
$$

The proof of the second part of the theorem run as follows.

$$
\frac{B_{k}^{(n)}-S}{B_{k-1}^{(n+1)}-S}=\frac{B_{k-1}^{(n+3)}-S-\frac{\left(B_{k-1}^{(n+3)}-B_{k-1}^{(n+1)}\right)\left(B_{k-1}^{(n+3)}-B_{k-1}^{(n+2)}\right)}{\left(B_{k-1}^{(n+3)}-B_{k-1}^{(n+1)}\right)-\left(B_{k-1}^{(n+2)}-B_{k-1}^{(n)}\right)}}{B_{k-1}^{(n+1)}-S},
$$

adding $S-S$ in each parenthesis and separating into two fractions we obtain

$$
\begin{aligned}
& \frac{B_{k}^{(n)}-S}{B_{k-1}^{(n+1)}-S}=\frac{B_{k-1}^{(n+3)}-S}{B_{k-1}^{(n+1)}-S}- \\
& \frac{\left[\left(B_{k-1}^{(n+3)}-S\right)-\left(B_{k-1}^{(n+1)}-S\right)\right]\left[\left(B_{k-1}^{(n+3)}-S\right)-\left(B_{k-1}^{(n+2)}-S\right)\right]}{\left\{\left[\left(B_{k-1}^{(n+3)}-S\right)-\left(B_{k-1}^{(n+1)}-S\right)\right]-\left[\left(B_{k-1}^{(n+2)}-S\right)-\left(B_{k-1}^{(n+1)}-S\right)\right]\right\}\left(B_{k-1}^{(n+1)}-S\right)} .
\end{aligned}
$$

Multiplying with $\frac{B_{k-1}^{(n+2)}-S}{B_{k-1}^{(n+2)}-S}$

$$
\begin{aligned}
\frac{B_{k}^{(n)}-S}{B_{k-1}^{(n)}-S} & =\frac{B_{k-1}^{(n+3)}-S}{B_{k-1}^{(n+2)}-S} \cdot \frac{B_{k-1}^{(n+2)}-S}{B_{k-1}^{(n+1)}-S}- \\
& -\frac{\left[\frac{B_{k-1}^{(n+3)}-S}{B_{k-1}^{(n+2)}-S}-\frac{B_{k-1}^{(n+1)}-S}{B_{k-1}^{(n+2)}-S}\right]\left[\frac{B_{k-1}^{(n+3)}-S}{B_{k-1}^{(n+2)}-S}-1\right]}{\left[\frac{B_{k-1}^{(n+3)}-S}{B_{k-1}^{(n+2)}-S}-\frac{B_{k-1}^{(n+1)}-S}{B_{k-1}^{(n+2)}-S}-1+\frac{B_{k-1}^{(n)}-S}{B_{k-1}^{(n+2)}-S}\right] \frac{B_{k-1}^{(n+1)}-S}{B_{k-1}^{(n+2)}-S}} .
\end{aligned}
$$


Taking the limit when $n$ tends to infinity

$$
\lim _{n \rightarrow \infty} \frac{B_{k}^{(n)}-S}{B_{k-1}^{(n+1)}-S}=b_{k}^{2}-\frac{\left(b_{k}-\frac{1}{b_{k}}\right)\left(b_{k}-1\right)}{\left(b_{k}-\frac{1}{b_{k}}-1+f r a c 1 b_{k}^{2}\right) \frac{1}{b_{k}}}
$$

from where it results

$$
\lim _{n \rightarrow \infty} \frac{B_{k}^{(n)}-S}{B_{k-1}^{(n+1)}-S}=b_{k}^{2}-b_{k}^{2}=0 .
$$

This completes the proof.

Our aim in the next section is to perform with our new algorithm an empirical study to test the capabilities of accelerating some numerical sequences arising in solving nonlinear equations for some test functions taken from [4], [5], [12], [18].

\section{NUMERICAL EXAMPLES}

In this section we present some numerical examples to illustrate the efficiency and the performance of the new proposed algorithm. The new acceleration method is applied to some numerical sequences arising in solving nonlinear equations that can be found in [4], [5], [12], [18]. To see the efficiency of the new proposed acceleration method we shall compare the sequence that arises when applying the new acceleration technique with the sequence that arises when applying Aitken's iterated $\Delta^{2}$ process. In the tables the numbers of exact decimals obtained at each step are displayed. All the numerical computations listed in the tables were done with Maple 13 using 39 digit floating point arithmetic.

Example 1. [4], [5] Test function: $f(x)=x^{3}+4 x^{2}-10$, which has a unique root $x^{*}=1.365230013414096845760806828981666078331 \ldots$. This equation can be rewritten into a fixed point problem by $g(x)=\frac{1}{2} \sqrt{10-x^{3}}$. To apply the new acceleration technique we shall take the initial guess $x_{0} \in\{1.2,1.3,1.4,1.5\}$. The results for Example 1 are listed in Table 1. 
Table 1

\begin{tabular}{|c|c|c|c|c|c|c|c|c|c|}
\hline \multicolumn{2}{|c|}{$\begin{array}{ll}\text { Initial guess } & \text { No. of iterations } \\
\end{array}$} & 1 & 2 & 3 & 4 & 5 & 6 & 7 & 8 \\
\hline \multirow{2}{*}{1.2} & $B_{k}^{(n)}$ & 2 & 5 & 9 & 13 & 20 & 26 & 31 & 38 \\
\hline & $A_{k}^{(n)}$ & 2 & 4 & 4 & 10 & 14 & 18 & 23 & 28 \\
\hline \multirow{2}{*}{1.3} & $B_{k}^{(n)}$ & 3 & 6 & 10 & 15 & 22 & 28 & 35 & 38 \\
\hline & $A_{k}^{(n)}$ & 2 & 4 & 8 & 12 & 16 & 21 & 26 & 32 \\
\hline \multirow{2}{*}{1.5} & $B_{k}^{(n)}$ & 2 & 4 & 9 & 12 & 20 & 25 & 32 & 38 \\
\hline & $A_{k}^{(n)}$ & 2 & 4 & 4 & 9 & 14 & 18 & 24 & 28 \\
\hline \multirow{2}{*}{1.6} & $B_{k}^{(n)}$ & 1 & 4 & 8 & 12 & 17 & 23 & 32 & 34 \\
\hline & $A_{k}^{(n)}$ & 1 & 3 & 4 & 8 & 13 & 16 & 24 & 25 \\
\hline
\end{tabular}

Example 2. [5] Test function $f(x)=x-3^{-x}=0$, which has a unique root $x^{*}=$ $0.5478086216540974464505754081510218503460 \ldots$ This equation can be rewritten as a fixed point problem by $g(x)=3^{-x}$. To apply the new acceleration technique we shall take the initial guess $x_{0} \in\{0.3,0.4,0.5,0.6\}$. The results for Example 2 are listed in Table 2.

Table 2

\begin{tabular}{|c|c|c|c|c|c|c|c|c|c|}
\hline \multicolumn{2}{|c|}{$\begin{array}{ll}\text { Initial guess } & \text { No. of iterations }\end{array}$} & 1 & 2 & 3 & 4 & 5 & 6 & 7 & 8 \\
\hline \multirow{2}{*}{0.3} & $B_{k}^{(n)}$ & 2 & 4 & 7 & 11 & 15 & 21 & 26 & 30 \\
\hline & $A_{k}^{(n)}$ & 1 & 3 & 5 & 8 & 12 & 16 & 19 & 23 \\
\hline \multirow{2}{*}{0.4} & $B_{k}^{(n)}$ & 2 & 4 & 8 & 11 & 16 & 23 & 28 & 32 \\
\hline & $A_{k}^{(n)}$ & 1 & 3 & 6 & 9 & 12 & 17 & 21 & 25 \\
\hline \multirow{2}{*}{0.5} & $B_{k}^{(n)}$ & 3 & 6 & 10 & 14 & 19 & 26 & 32 & 37 \\
\hline & $A_{k}^{(n)}$ & 2 & 5 & 8 & 12 & 16 & 21 & 25 & 30 \\
\hline \multirow{2}{*}{0.6} & $B_{k}^{(n)}$ & 3 & 6 & 10 & 14 & 19 & 27 & 31 & 37 \\
\hline & $A_{k}^{(n)}$ & 2 & 5 & 8 & 12 & 16 & 21 & 25 & 29 \\
\hline
\end{tabular}

Example 3. [4] Test function $f(x)=\cos x-x=0$, which has a unique root $x^{*}=0.7390851332151606416553120876738734040134 \ldots$. This equation can be rewritten into a fixed point problem by $g(x)=\cos x$. To apply the new acceleration technique we shall take the initial guess $x_{0} \in\{0.5,0.7,0.8,0.9\}$. The results for Example refe 3 are listed in Table 3. 
Table 3

\begin{tabular}{|c|c|c|c|c|c|c|c|c|c|}
\hline \multicolumn{2}{|c|}{$\begin{array}{ll}\text { Initial guess } & \text { No. of iterations } \\
\end{array}$} & 1 & 2 & 3 & 4 & 5 & 6 & 7 & 8 \\
\hline \multirow{2}{*}{0.5} & $B_{k}^{(n)}$ & 1 & 3 & 5 & 8 & 11 & 14 & 18 & 23 \\
\hline & $A_{k}^{(n)}$ & 2 & 2 & 3 & 6 & 8 & 11 & 16 & 17 \\
\hline \multirow{2}{*}{0.7} & $B_{k}^{(n)}$ & 3 & 5 & 8 & 12 & 15 & 19 & 25 & 30 \\
\hline & $A_{k}^{(n)}$ & 2 & 5 & 7 & 10 & 14 & 16 & 22 & 23 \\
\hline \multirow{2}{*}{0.8} & $B_{k}^{(n)}$ & 3 & 4 & 7 & 11 & 13 & 18 & 22 & 28 \\
\hline & $A_{k}^{(n)}$ & 2 & 3 & 7 & 8 & 11 & 15 & 19 & 23 \\
\hline \multirow{2}{*}{0.9} & $B_{k}^{(n)}$ & 1 & 2 & 5 & 9 & 11 & 15 & 19 & 24 \\
\hline & $A_{k}^{(n)}$ & 2 & 3 & 4 & 6 & 9 & 12 & 16 & 19 \\
\hline
\end{tabular}

Example 4. [12] Test function $f(x)=(x+2) e^{x}-1=0$, which has a unique root $x^{*}=-0.4428544010023885831413279999993368197163 \ldots$. This equation can be rewritten into a fixed point problem by $g(x)=-\ln (x+2)$. To apply the new acceleration technique we shall take the initial guess $x_{0} \in\{-0.2,-0.4,-0.5,-0.6\}$. The results for Example 4 are listed in Table 4.

Table 4

\begin{tabular}{|c|c|c|c|c|c|c|c|c|c|}
\hline \multicolumn{2}{|c|}{$\begin{array}{ll}\text { Nnitial guess } & \text { No. of iterations } \\
\end{array}$} & 1 & 2 & 3 & 4 & 5 & 6 & 7 & 8 \\
\hline \multirow{2}{*}{-0.2} & $B_{k}^{(n)}$ & 2 & 4 & 7 & 8 & 15 & 19 & 23 & 32 \\
\hline & $A_{k}^{(n)}$ & 1 & 3 & 6 & 6 & 11 & 15 & 19 & 23 \\
\hline \multirow{2}{*}{-0.4} & $B_{k}^{(n)}$ & 3 & 6 & 10 & 14 & 19 & 25 & 31 & 38 \\
\hline & $A_{k}^{(n)}$ & 3 & 6 & 9 & 12 & 16 & 20 & 23 & 29 \\
\hline \multirow{2}{*}{-0.5} & $B_{k}^{(n)}$ & 2 & 6 & 10 & 13 & 18 & 23 & 30 & 37 \\
\hline & $A_{k}^{(n)}$ & 3 & 5 & 8 & 11 & 15 & 19 & 23 & 23 \\
\hline \multirow{2}{*}{-0.6} & $B_{k}^{(n)}$ & 2 & 5 & 8 & 11 & 15 & 21 & 23 & 33 \\
\hline & $A_{k}^{(n)}$ & 2 & 4 & 7 & 9 & 12 & 16 & 20 & 21 \\
\hline
\end{tabular}

Example 5. [18] Test function $f(x)=x^{3}-2 x^{2}-5=0$, which has a unique root $x^{*}=2.690647448028613750350788882676806151802 \ldots$. This equation can be rewritten into a fixed point problem by $g(x)=\sqrt[3]{2 x^{2}+5}$. To apply the new acceleration techniqu e we shall take the initial guess $x_{0} \in\{2.3,2.5,2.8,3.0\}$. The results for Example 5are listed in Table 5. 
Table 5

\begin{tabular}{|c|c|c|c|c|c|c|c|c|c|}
\hline \multicolumn{2}{|c|}{$\begin{array}{ll}\text { Initial guess } & \text { No. of iterations } \\
\end{array}$} & 1 & 2 & 3 & 4 & 5 & 6 & 7 & 8 \\
\hline \multirow{2}{*}{2.3} & $B_{k}^{(n)}$ & 3 & 6 & 9 & 12 & 16 & 21 & 28 & 36 \\
\hline & $A_{k}^{(n)}$ & 1 & 5 & 8 & 9 & 11 & 15 & 18 & 23 \\
\hline \multirow{2}{*}{2.5} & $B_{k}^{(n)}$ & 4 & 6 & 9 & 12 & 18 & 24 & 30 & 38 \\
\hline & $A_{k}^{(n)}$ & 3 & 5 & 8 & 8 & 13 & 17 & 21 & 27 \\
\hline \multirow{2}{*}{2.8} & $B_{k}^{(n)}$ & 6 & 7 & 9 & 14 & 20 & 26 & 32 & 38 \\
\hline & $A_{k}^{(n)}$ & 5 & 5 & 7 & 13 & 14 & 20 & 23 & 29 \\
\hline \multirow{2}{*}{3.0} & $B_{k}^{(n)}$ & 3 & 6 & 7 & 11 & 17 & 24 & 29 & 36 \\
\hline & $A_{k}^{(n)}$ & 3 & 5 & 5 & 10 & 12 & 15 & 20 & 24 \\
\hline
\end{tabular}

\section{Conclusions}

In this paper we presented a new acceleration method inspired from one of the derivation of Aitken's iterated $\Delta^{2}$ process given by the expression (2.6). To see the efficiency of the new acceleration method we performed a comparison between the sequence that arises when applying the new acceleration technique and the sequence that arises when applying Aitken's iterated $\Delta^{2}$ process in order to see which one has the best convergence speed. We have done studies on twenty test functions, we here presented only five of them. For all test functions, that we studied, the new acceleration method improves significantly the convergence speed of a scalar sequence.

\section{REFERENCES}

[1] A. C. Aitken, “On Bernoulli's numerical solution of algebraic equations," Proceedings Royal Soc. Edinburgh, vol. 46, pp. 289-305, 1926.

[2] G. A. Baker and P. Graves-Morris, Padé approximants. 2nd ed., ser. Encyclopedia of Mathematics and Its Applications. Cambridge: Cambridge University Press, 1996, vol. 59.

[3] V. Berinde, Iterative approximation of fixed points. 2nd revised and enlarged ed., ser. Lecture Notes in Mathematics. Berlin: Springer, 2007, vol. 1912.

[4] V. Berinde and O. Bumbariu, "Empirical study of a padé type accelerating method of picard iteration," Creat. Math. Inform., vol. 19, no. 2, pp. 149-159, 2010.

[5] J. Biazar and A. Amirteimoori, "An improvement to the fixed point iterative method," Appl. Math. Comput., vol. 182, no. 1, pp. 567-571, 2006.

[6] C. Brezinski, "Accélération du suites à convergence logarithmique," C. R. Acad. Sci., Paris, Sér. A, vol. 273, pp. 727-730, 1971.

[7] C. Brezinski, Accélération de la convergence en analyse numérique, ser. Lecture Notes in Mathematics. Berlin-Heidelberg-New York: Springer-Verlag, 1977, vol. 584.

[8] C. Brezinski, Algorithmes d'accélération de la convergence. Étude numérique, ser. Collection Langages et Algorithmes de l'Informatique. Paris: Editions Technip., 1978.

[9] C. Brezinski, "Convergence acceleration during the 20th century," J. Comput. Appl. Math., vol. 122 , no. $1-2$, pp. $1-21,2000$. 
[10] F. Cordellier, "Sur la régularité des procédés $\delta^{2}$ d'Aitken et $W$ de Lubkin," in Pade approximation and its applications. Proc. Conf., ser. Lect. Notes Math., vol. 765. Antwerp: Springer, 1979, pp. 20-35.

[11] J. E. Drummond, "Summing a common type of slowly convergent series of positive terms," $J$. Aust. Math. Soc., Ser. B, vol. 19, pp. 416-421, 1976.

[12] Y. Ham and C. Chun, "A fifth-order iterative method for solving nonlinear equations," Appl. Math. Comput., vol. 194, no. 1, pp. 287-290, 2007.

[13] M. J. Jamieson and T. H. O'Beirne, "A note on a generalization of Aitken's $\Delta^{2}$ transformation," J. Phys. B, vol. 11, pp. 31-35, 1978.

[14] D. Levin, "Development of non-linear transformations for improving convergence of sequences," Int. J. Computer Math., vol. 3, pp. 371-388, 1973.

[15] S. Lubkin, "A method of summing infinite series," J. Res. Nat. Bur. Stand., vol. 48, pp. 228-254, 1952.

[16] A. J. MacLeod, “Acceleration of vector sequences by multi-dimensional $\Delta^{2}$ methods," Commun. Appl. Numer. Methods, vol. 2, pp. 385-392, 1986.

[17] Şt. Măruşter, Metode numerice în rezolvar ea ecuaţiilor neliniare. Bucuresti: Editura Tehnica, 1981.

[18] M. A. Noor, K. I. Noor, W. A. Khan, and F. Ahmad, "On iterative methods for nonlinear equations," Appl. Math. Comput., vol. 183, no. 1, pp. 128-133, 2006.

[19] J. M. Ortega and W. C. Rheinboldt, Iterative solution of nonlinear equations in several variables, ser. Computer Science and Applied Mathematics. New York-London: Academic Press, 1970.

[20] D. Shanks, "Non-linear transformations of divergent and slowly convergent sequences," J. Math. Phys., Mass. Inst. Techn., vol. 34, pp. 1-42, 1955.

[21] D. A. Smith and W. F. Ford, "Numerical comparisons of nonlinear convergence accelerators," Math. Comput., vol. 38, pp. 481-499, 1982.

[22] R. R. Tucker, "The $\delta^{2}$-process and related topies," Pac. J. Math., vol. 22, pp. 349-359, 1967.

[23] R. R. Tucker, “The $\delta^{2}$-process and related topics. ii." Pac. J. Math., vol. 28, pp. 455-463, 1969.

[24] G. Walz, Asymptotics and extrapolation, ser. Mathematical Research. Berlin: Akademie Verlag, 1996, vol. 88.

[25] E. J. Weniger, "Nonlinear sequence transformations for the acceleration of convergence and the summation of divergent series," Comput. Phys. Rep., vol. 10, pp. 189-371, 1989.

[26] J. Wimp, Sequence transformations and their applications, ser. Mathematics in Science and Engineering. New York - London: Academic Press, 1981, vol. 154.

[27] P. Wynn, "On a device for computing the $e_{m}\left(S_{n}\right)$ transformation," Math. Tables Aids Comput., vol. 10, pp. 91-96, 1956.

\section{Author's address}

\section{Oana Bumbariu}

North University of Baia Mare, Department of Mathematics and Computer Science, 76 Victoriei, 430122 Baia Mare, Romania

E-mail address: oanabumbariu@yahoo.com 